\title{
Anomalous optical absorption in overdoped cuprates near the charge-ordering instability
}

\author{
S. Caprara ${ }^{1}$, C. Di Castro ${ }^{1}$, S. Fratini ${ }^{1,2}$, and M. Grilli ${ }^{1}$ \\ ${ }^{1}$ Istituto Nazionale per la Fisica della Materia - Unità di Roma 1, and Dipartimento di Fisica - Università di Roma "La \\ Sapienza", Piazzale Aldo Moro 2, I-00185 Roma, Italy \\ ${ }^{2}$ Departamento de Teoria de la Materia Condensada - Instituto de Ciencia de Materiales de Madrid (CSIC) Cantoblanco, \\ E-28049 Madrid, Spain
}

\begin{abstract}
We propose an interpretation for the hump observed in the optical conductivity at or below a few hundreds of $\mathrm{cm}^{-1}$, in overdoped cuprates like the electron-doped $\mathrm{Nd}_{2-\mathrm{x}} \mathrm{Ce}_{\mathrm{x}} \mathrm{CuO}_{4-\mathrm{y}}$ at $x \gtrsim 0.15$ and the hole-doped $\mathrm{Bi}_{2} \mathrm{Sr}_{2} \mathrm{CuO}_{6}$ and $\mathrm{La}_{2-\mathrm{x}} \mathrm{Sr}_{\mathrm{x}} \mathrm{CuO}_{4}$. This interpretation is based on the direct excitation of charge collective modes, which become nearly critical in the proximity to a charge-ordering instability. The nearly critical character of these excitations entails a peculiar temperature dependence and a pseudo-scaling form of the lineshapes, which are in agreement with the experimental data.
\end{abstract}

PACS: 74.72.-h, 71.45.Lr, 74.25.Gz, 72.10.Di

The extension of optical conductivity experiments to very low frequencies in the range of the very far infrared $\left(\omega \gtrsim 10 \mathrm{~cm}^{-1}\right)$ has uncovered unexpected intriguing features. In particular recent measurements of $\sigma(\omega)$ on a slightly overdoped $\mathrm{Bi}_{2} \mathrm{Sr}_{2} \mathrm{CuO}_{6}(\mathrm{Bi} 2201)$ sample [1] revealed a strong absorption peak at a few hundreds of $\mathrm{cm}^{-1}$ narrowing and softening upon decreasing the temperature and well separated from a much weaker and narrower Drude term. The extrapolation to zero frequency of this latter spectral contribution matches well with the d.c. value $\sigma(\omega=0, T)$ as obtained from transport data. Althought this is the only case where a separate narrow Drude term is directly observed, there are other cases both in hole- and electron-doped cuprates, like $\mathrm{La}_{2-\mathrm{x}} \mathrm{Sr}_{\mathrm{x}} \mathrm{CuO}_{4}$ ( $\mathrm{LSCO}, \mathrm{x}=0.184,0.22$ ) [2] and $\mathrm{Nd}_{2-\mathrm{x}} \mathrm{Ce}_{\mathrm{x}} \mathrm{CuO}_{4-\mathrm{y}}$ at $x \gtrsim 0.15$ (NCCO) [3, ], where a strongly temperature dependent peak at a finite frequency $\Omega_{\text {Max }}$ is observed. Moreover, in LSCO, for $\omega<\Omega_{\text {Max }}, \sigma(\omega)$ decreases and moves away from the substantially higher d.c. value. This indicates that a Drude term, which is beyond the lowest measured frequency, must intervene to restore a high value of $\sigma$ consistently with the zero-frequency data and with the marked metallic character of these overdoped systems $[5]$.

We find that the (direct or indirect) observation of these two separated spectral features (the peak at $\Omega_{\text {Max }} \lesssim 100-200 \mathrm{~cm}^{-1}$ and the narrow Drude term) calls both for an extensive experimental investigation at very low frequencies and a careful reanalysis of the theoretical framework. In this latter regard, the presence of two well separated features, with different temperature dependence (at least in Bi2201) shows that a singlefluid picture, with a strongly frequency-dependent inverse scattering time $1 / \tau(\omega)$ is inadequate. In particular the above experiments show that (at least in some cases) the broad, slowly decaying $(\sim 1 / \omega)$ optical absorption cannot be attributed to strongly interacting carriers responsible for an anomalous Drude peak at zero frequency, and rather arises from finite-frequency excitations clearly distinct from much less interacting (and not anomalous) Drude carriers. The interpretation of optical spectra at somewhat higher frequency $\omega>200-300 \mathrm{~cm}^{-1}$, in terms of quasiparticles (QP's) scattered by (spin) collective modes (CM's) [6.7], seems hardly viable to account for the peculiar behavior of $\sigma(\omega)$ in the above systems at $\omega \lesssim 100-200 \mathrm{~cm}^{-1}$. In particular, as we checked (see below), and according to previous results [8,9], overdamped modes (as the spin waves would be in the markedly metallic optimally and over-doped materials) cannot produce the pronounced Drude-dip-hump structure of the spectra.

We propose, instead, that the finite-frequency peak arises from the absorption due to the excitation of pairs of CM's directly coupled to the electromagnetic (e.m.) field. These CM's are i) abundant at low frequency and ii) strongly temperature dependent, because they are critical modes of a (hidden) criticality ending at $T=0$ into a quantum critical point (QCP) associated to a chargeordering $(\mathrm{CO})$ instability [?] at a critical doping $x_{c}$ close to optimal doping 13. We focus on the low-frequency optical response of systems like the above $\mathrm{NCCO}$ and Bi2201, which are in the optimally or slightly over-doped regions, where a simpler description in terms of QP's scattered by the (nearly) critical charge fluctuations $\rho_{\mathbf{q}}$, associated with the nearby critical line $T_{C O}(x) \approx T^{*}$, is reasonable. We introduce the phenomenological Hamiltonian

$$
\mathcal{H}=\sum_{\mathbf{k}, \sigma} \xi_{\mathbf{k}} c_{\mathbf{k}, \sigma}^{\dagger} c_{\mathbf{k}, \sigma}+g \sum_{\mathbf{k}, \mathbf{q}, \sigma} c_{\mathbf{k}+\mathbf{q}, \sigma}^{\dagger} c_{\mathbf{k}, \sigma} \rho_{\mathbf{q}}
$$

where $c_{\mathbf{k}, \sigma}^{(\dagger)}$ are the QP operators, $\xi_{\mathbf{k}}$ is the QP band and $g$ is the QP-CM coupling constant. While the actual form of $\xi_{\mathrm{k}}$ is of minor importance, the form of the CM (retarded) propagator is quite relevant,

$$
D^{R}(\mathbf{q}, \omega)=\left[\Gamma+\nu\left|\mathbf{q}-\mathbf{q}_{c}\right|^{2}-\mathrm{i} \omega\right]^{-1} .
$$

Such a form, which pertains to a Gaussian QCP with dynamical critical index $z=2$, was previously derived 
within a microscopic Hubbard-Holstein model with longrange Coulomb forces [?], but is rather generic, as it can be obtained from other microscopic models with, e.g., antiferromagnetic [14] or excitonic 15] interactions. The "mass term" $\Gamma$ determines the proximity to the $\mathrm{CO}$ instability and plays a crucial role since its peculiar temperature dependence $\Gamma \approx \max \left[a\left(x-x_{c}\right), b T\right]$ is a signature of quantum critical fluctuations 16] and has observable consequences in the optical spectra. Taking all lengths in units of the lattice spacing, the CM "bandwidth" is controlled by the energy scale $\nu$ (of the order of the QP Fermy energy $E_{F}$ ), which is the inverse of a characteristic relaxation time. The wave vector $\mathbf{q}_{c}$ sets the wavelenght of the most critical charge density fluctuations, $\lambda_{C O} \sim\left|\mathbf{q}_{c}\right|^{-1}$.

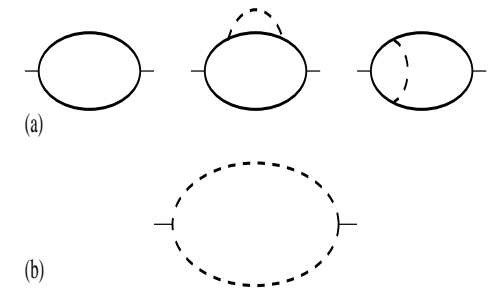

FIG. 1. (a) QP (full line) response to the e.m. field, dressed by nearly critical CM's (dashed line) at the lowest order in perturbation theory. (b) CM (dashed line) response to the e.m. field, resulting from the phenomenological coupling (3).

Within the model (11,2) we calculate the optical conductivity arising from (i) the response of the fermionic QP's which are scattered by the (nearly) critical CM's [Fig. 1(a)] and (ii) the response of the CM's themselves [Fig. 1(b)], which couple to the e.m. vector potential via an effective phenomenological interaction

$$
\mathcal{H}_{A \rho}=\bar{g} \sum_{\mathbf{Q}, \mathbf{q}} \rho_{\frac{\mathbf{q}+\mathbf{Q}}{2}} \rho_{\frac{-\mathbf{q}+\mathbf{Q}}{2}} \mathbf{A}_{\mathbf{Q}} \cdot \mathbf{w}(\mathbf{q}, \mathbf{Q})
$$

where $\mathbf{w}(\mathbf{q}, \mathbf{Q})$ is a vectorial matrix element. The coupling term (3) deserves some discussion. We are primarily interested in the effects of nearly critical charge fluctuations, which in the $\mathrm{CO}$ scenario occur at finite wave vectors $\mathbf{q} \approx \mathbf{q}_{c}$. Therefore the e.m. excitation of a single $\mathrm{CM}$ at $|\mathbf{q}| \approx 0$ is of little interest at low energy and we discard it. On the other hand, the simultaneous excitation of two CM's with momenta $\mathbf{q} \approx \pm \mathbf{q}_{c}$ [Fig. 1(b)] becomes relevant. In this case the actual expression of $\mathbf{w}(\mathbf{q}, \mathbf{Q})$ is immaterial since for $\mathbf{q} \approx \pm \mathbf{q}_{c}$ this vertex only provides a numerical coefficient, which can be reabsorbed in $\bar{g}$. Of course, these processes can only occur in the presence of an effective coupling between the transverse e.m. field and the longitudinal charge CM's, which, however, is not problematic. First of all, the charge modulation superimposed to the uniform ionic background can create a dipolar moment coupling to the e.m. field [17]. Secondly extrinsic disorder (from impurities) and/or intrinsic disorder (possibly from a nearly static short-range CO texture) can render the very concept of longitudinal and transverse excitations meaningless. Finally, and most importantly, the direct e.m. excitation of pairs of longitudinal optical phonons via quadrupolar coupling or via anharmonic effects is a quite well-known phenomenon, which can even acquire a dominant spectral weight close to (structural) phase transitions [18].

The QP response, including the standard self-energy and vertex corrections due to the CM's, and the direct excitation of the CM's, act as separate channels in parallel, and are represented by the diagrams of Fig. 1(a) and (b) respectively. For $\omega \ll E_{F}$, the QP and CM lowestorder corrections to the current-current correlation functions have the common form

$$
\chi_{Q P, C M}=\frac{2 C_{Q P, C M}}{\pi \bar{\Omega}}\left[\phi_{Q P, C M}^{\prime}\left(\frac{\omega}{\bar{\Omega}}\right)+\mathrm{i} \phi_{Q P, C M}^{\prime \prime}\left(\frac{\omega}{\bar{\Omega}}\right)\right],
$$

where $C_{Q P, C M}$ are constants, and $\bar{\Omega} \sim \Gamma$. The imaginary parts $\phi^{\prime \prime}$ are calculated evaluating the corresponding expressions of the last two diagrams of Fig. 1(a) at $T=0$, while maintaining the relevant temperature dependence of $\bar{\Omega} \sim \Gamma$. We numerically checked that this approximation induces minor quantitative corrections at temperatures and frequencies of interest here, while allowing for the explict derivation of the final expressions. The real parts $\phi^{\prime}$ are obtained by a Kramers-Krönig transformation, yielding, for $z>0$,

$$
\begin{aligned}
\phi_{Q P}^{\prime \prime}(z) & =-\left[\frac{1}{z^{2}} \arctan z-\frac{1}{z}+\frac{1}{2 z} \ln \left(1+z^{2}\right)\right], \\
\phi_{Q P}^{\prime}(z) & =-\left[\frac{1}{2 z} \arctan z+\frac{1}{4 z^{2}} \ln \left(1+z^{2}\right)\right] \\
\phi_{C M}^{\prime \prime}(z) & =\frac{1}{z^{2}+4}\left[\arctan z-\frac{z^{2}+2}{2 z} \ln \left(1+z^{2}\right)\right], \\
\phi_{C M}^{\prime}(z) & =-\frac{1}{z^{2}+4}\left[\frac{z^{2}+2}{z} \arctan z+\frac{1}{2} \ln \left(1+z^{2}\right)\right] .
\end{aligned}
$$

The contribution of the CM's to the complex conductivity is obtained by directly inserting the corresponding current-current response function from the diagram in Fig. 1(b) into the Kubo formula yielding

$$
\sigma_{C M}(\omega)=\frac{\mathrm{i} \chi_{C M}(\omega)}{\omega} .
$$

On the other hand, it is well known 19] that $\chi_{Q P}$, which is the lowest-order correction to the optical response of a perfect metal, provides a valid expression for $\sigma(\omega)$ in the high-frequency regime only $(\omega \tau \gg 1)$. This difficulty is customarily overcome by expressing the the QP contribution to the conductivity in terms of a complex memory function $M$ 19. 


$$
\sigma_{Q P}(\omega)=\frac{\omega_{p}^{2}}{4 \pi} \frac{1}{M_{Q P}(\omega, T)-\mathrm{i} \omega} .
$$

A perturbative approach to $M$ is now viable, even at low frequencies leading to $M_{Q P} \approx i \omega 4 \pi \chi_{Q P} / \omega_{p}^{2}$. For $\sigma_{Q P}$ this corresponds to a RPA resummation of the diagrams in Fig. 1(a). Here $\omega_{p}$ is the plasma frequency, while $\operatorname{Re} M$ and $-\operatorname{Im} M / \omega$ play the role of the inverse scattering time, and "mass renormalization" (which reduces the low-frequency absorption), respectively.

The total absorption arises from the parallel of the two separate contributions given in Eqs. (9), (10) $\sigma=$ $\sigma_{Q P}+\sigma_{C M}$. A similar two-component conductivity was discussed within a one-dimensional Kondo-lattice model [17], where, however, no critical behavior was present. Within this scheme, the scattering of the QP's with impurities, with a scattering time $\tau_{i m p}$, is in series with the scattering of the QP's with the CM's and, according to the Matthiessen's rule, is accounted for by taking $M_{Q P} \rightarrow M_{Q P}+\tau_{i m p}^{-1}$.

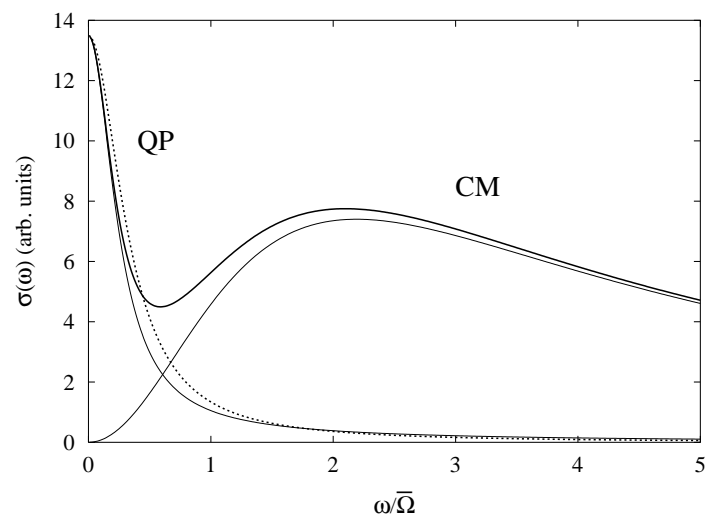

FIG. 2. Thick line: typical optical absorption from collective-modes [CM, eq. (9)] and quasiparticles [QP, eq. (10)], including impurity scattering with $\tau_{i m p}^{-1}=\bar{\Omega} / 3$. The separate contributions are shown as thin lines. An ordinary Drude peak with the same $\tau_{i m p}^{-1}$ is shown for comparison (dotted line). Notice the depletion of spectral weight at $\omega \lesssim \bar{\Omega}$ due to the QP-CM scattering.

A typical optical spectrum resulting from our calculations is displayed in Fig. 2. In the absence of disorder, the QP contribution consists of a (nearly) delta-like absorption at zero frequency [essentially arising from the first diagram of Fig. 1(a)] plus a long tail which monotonically decreases with $\omega$ according to Eqs. (5) and (6). In agreement with previous analyses 8.5] this second contribution has a weight proportional to the QPCM coupling $g^{2}$, while its width is controlled by $\bar{\Omega}$. Impurity scattering gives the zero-frequency peak a finite width, which, mostly due to mass-renormalization effects (the ImM contribution), is less than the expected Drude value $\tau_{i m p}^{-1}$ (see dotted line in Fig. 2), indicating that the QP-CM scattering transfers some spectral weight from the region $\omega \lesssim \tau_{i m p}^{-1}$ to the region $\omega \sim \bar{\Omega}$.
Roughly speaking this occurs because the QP's dressed with the CM's acquire spectral weight around the typical CM frequencies (and in some sense also a partial CM character) thereby becoming less sensitive to the singleparticle scattering due to impurities. As a result, it turns out that the coupling with the CM's narrows the Drude peak for $\omega \lesssim \tau_{i m p}^{-1}$ and the system appears somewhat cleaner than it would be in the absence of QP-CM coupling. However, even taking a very large $g^{2}$, we fail in producing a non-monotonic behavior with maxima at finite frequency. Moreover, we see that for all reasonable choices of the parameters, the broad tail is very weak compared to the large finite-frequency absorption seen in the experiments. By no means one can reconcile the low and narrow Drude peak with the higher and broader hump within the QP component only. The contribution from the diagram in Fig. 1(b) is therefore crucial to reproduce the experimental hump and supports the introduction of the phenomenological interaction (3). Indeed the convolution of two overdamped CM's having the critical form of Eq. (2), leads to an absorption with a (broad) maximum at $\omega=\Omega_{\text {Max }}=2 \bar{\Omega} \sim 2 \Gamma$. Theoretically, this quantity should scale proportionally to $T$ at high temperature, in the quantum critical regime, and saturate to a constant value $\propto\left(x-x_{c}\right)$ in the low-temperature quantum disordered regime, as ruled by the proximity to a QCP. The critical character of the propagator (2) entails a scaling form in the frequency dependence of $\chi(\omega)=$ const $\times \phi\left(\omega / \Omega_{\text {Max }}\right) / \Omega_{\text {Max }}$. As a consequence, the corresponding finite-frequency features in $\sigma(\omega)$ display a scaling behavior, when plotted as a function of $\omega / \Omega_{\text {Max }}$ (see Fig. 3) [20].

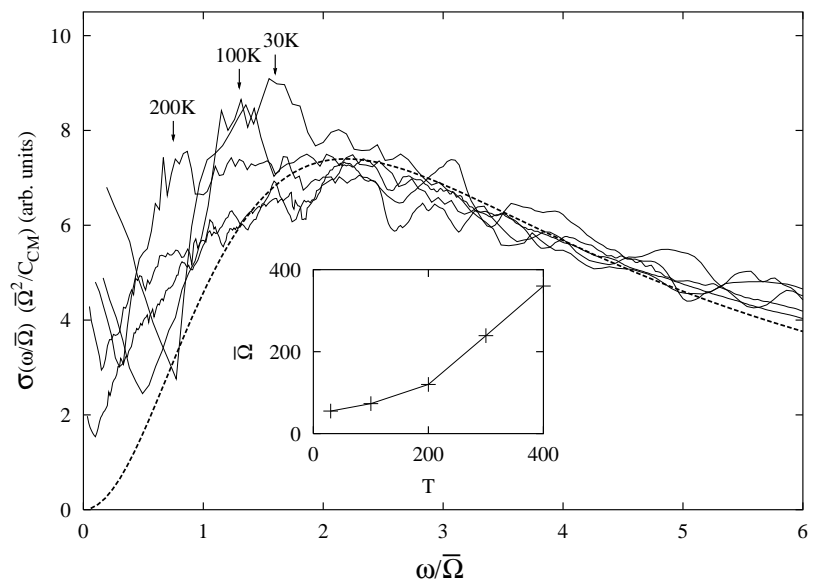

FIG. 3. Scaled optical absorption $\sigma(\omega / \bar{\Omega})\left(\bar{\Omega} / \bar{\omega}_{p}\right)^{2}$ for slightly overdoped Bi2201 at different temperatures. The absorption peaks all fall on a single theoretical curve (dashed), given by Eq. (6). The deviations from the scaling curve in the low frequency part come from the Drude contribution (data courtesy of S. Lupi et al.). The inset shows the behavior of the fit parameter $\bar{\Omega}$ vs $T$, characteristic of the crossover from a quantum critical to a quantum disordered regime (see text). 
The peculiar $\omega$ and $T$ dependence of $\sigma$ described above is indeed seen in the optical spectra of the electron-doped NCCO at $x \gtrsim 0.15$ 3. 4 and of the hole-doped Bi2201 [1] in the non-superconducting phase. Despite the limited amount of available data, we find quite encouraging that our approach is in remarkable agreement with experiments whenever a comparison is possible (i.e. in experiments carried out down to very low frequencies $\left.\omega \gtrsim 10 \mathrm{~cm}^{-1}\right)$. Specifically, we use our theory to fit the spectra of Bi2201, reported in Ref. [1]. According to the above discussion, we analyze the Drude part and the anomalous hump separately. The latter can be fitted adjusting the strength of the (e.m. field)-CM coupling $\bar{g}$ [more exactly the constant $C_{C M}$ in Eq. (4)] determining the weight of the absorption from CM's and the CM characteristic frequency scale $\bar{\Omega} \sim \Gamma$. As it can be seen from Fig. 3, where we plot the experimental and theoretical curves in the scaled variable $\omega / \bar{\Omega}$ the scaling collapse of the curves into a single curve and the fit of the spectra at different temperatures is satisfactory. A similar agreement is found in the case of the NCCO and LSCO data of Refs. [4,2] respectively.

A direct inspection of the non-rescaled data in Fig. 3 of Ref. [1] shows the presence around $100 \mathrm{~cm}^{-1}$ of a narrow feature (likely of phononic origin) which narrows but does not shift upon lowering the temperature. This extrinsic feature (marked by vertical arrows in Fig. 3) obviously does not possess any scaling character, which explains the deviations from the universal curve in the low-frequency region.

It is quite remarkable, that $\bar{\Omega}=\Omega_{\text {Max }} / 2$ as obtained from the fits and reported in the inset of Fig. 3, is linear in temperature and then saturates at a finite value at low temperatures as expected for $\Gamma$ from quantum criticality. A similar quantum-critical dependence has recently been observed by ARPES in the scattering rate of QP's in optimally-doped Bi2212 [21], indicating the still not understood but remarkably general character of this behavior.

We now turn our attention to the small and narrow zero-frequency peak. Setting the CM-QP coupling to zero, as shown in Ref. [1], this peak could be easily fitted as an ordinary Drude peak with two parameters, the plasma frequency $\omega_{p}$, which provides the intensity, and the scattering rate $\tau_{i m p}^{-1} \approx 30-50 \mathrm{~cm}^{-1}$, which controls the width. These values for $\tau_{i m p}^{-1}$ are fairly small for such chemically complex compounds. However, according to the discussion of Fig. 2, the introduction of a moderate QP-CM coupling tends to reduce the width of the Drude peak thereby rendering the observed Drude width compatible with $\tau_{i m p}^{-1}$ 's as large as $100-200 \mathrm{~cm}^{-1}$.

The comparison between theory and experiments is only meaningful at low frequency, since our theory is based on a general analysis of quantum critical phe- nomena, which fails in describing the non-critical highfrequency (mid-infrared, charge-transfer,...) parts of the spectra. Nevertheless, the (approximate) scaling form and temperature dependence of $\sigma(\omega)$ are nontrivial robust features of our results. We notice that the pseudoscaling properties of the softening of the finite-frequency feature upon reducing the temperature is hardly interpreted within other (more traditional) mechanisms, like the binding due to impurity states [4], and indicates that $T$ is the only relevant energy scale, determining both the position and the shape of the "hump" spectral feature, in a broad temperature range. At the moment, we are not aware of any well-established alternative explanation, other than the direct absorption from nearly critical CM's. This is particularly important, not only because it provides an explanation for the spectral features of specific compounds, but also, and most importantly, because it supports the peculiar role of critical charge fluctuations in ruling the physical properties of the cuprates.

Acknowledgments. We acknowledge stimulating discussions with P. Calvani, M. Capizzi, C. Castellani, and S. Lupi, whom we also thank for providing us the data of the optical conductivity in Bi2201.

[1] S. Lupi, et al., Phys. Rev. B 62, 12418 (2000).

[2] T. Startseva, et al., Physica C 321, 135 (1999).

[3] S. Lupi, et al., Phys. Rev. Lett. 83, 4852 (1999).

[4] E. J. Singley, et al., cond-mat/0103480.

[5] On the other hand, in NCCO and in other hole-doped compounds like $\mathrm{Tl}_{2} \mathrm{Ba}_{2} \mathrm{CuO}_{6+\mathrm{x}}$ [A. V. Puchkov, et al., Phys. Rev. B 51, 3312] or $\mathrm{Tl}_{2} \mathrm{Ba}_{2} \mathrm{CaCu}_{2} \mathrm{O}_{8}$ [C. M. Foster, et al. Solid State Commun. 76, 651 (1990)] the Drude peak at zero frequency should have a value lower than the peak and low-frequency experiments are needed to see whether this term is well separated (like in Bi2201) or not from the finite-frequency hump.

[6] J. P. Carbotte, E. Schachinger, and D. N. Basov, Nature 401, 354 (1999).

[7] Ar. Abanov, A. Chubukov, and J. Schmalian, Phys. Rev. B 63, 180510(R) (2001).

[8] For a review see, e.g., A. V. Puchkov, D. N. Basov, and T. Timusk, J. Phys.: Condens. Matter 8, 10046 (1996).

[9] H. J. Kaufmann, E. G. Maksimov, and E. K. H. Salje, cond-mat/9805108.

[10] C. Castellani, C. Di Castro, and M. Grilli, Phys. Rev. Lett. 75, 4650 (1995).

[11] A. Perali, et al., Phys. Rev. B 54, 16216 (1996).

[12] S. Andergassen, et al., Phys. Rev. Lett. 87, 056401 (2001).

[13] For a summary of evidences of a QCP near optimal doping see, e.g., C. Castellani, C. Di Castro, and M. Grilli, Z. für Physik 103, 137 (1997); C. M. Varma, Phys. 
Rev. Lett. 83, 3538 (1999) and references therein; C. Panagopoulos, et al., cond-mat/0007158; D. L. Feng, et al. Science 289, 277 (2000); M. Gutmann, E. S. Božin, and S. J. L. Billinge, cond-mat/0009141.

[14] A. J. Millis, H. Monien, and D. Pines, Phys. Rev. B 42, 167 (1990).

[15] F. Becca, F. Bucci, and M. Grilli, Phys. Rev. B 57, 4382 (1998).

[16] This behavior is an approximate way to interpolate between the two asymptotic behaviors $\Gamma \approx a\left(x-x_{c}\right)$ or $\Gamma \approx b T$ (with $a$ and $b$ non-universal constants) characterizing the quantum disordered and the quantum critical regimes respectively.

[17] V. J. Emery and S. A. Kivelson, Phys. Rev. Lett. 71, 3701 (1993).

[18] B. Donovan and J. F. Angress, Lattice vibrations, Chapman and Hall LTD., London (1971).

[19] W. Götze and P. Wölfle, Phys. Rev. B 6, 1226 (1972).

[20] On the other hand, the presence of the $\mathrm{i} \omega$ term in the denominator of Eq. (10) prevents the $\sigma_{Q P}$ part of the conductivity to acquire a fully scaling dependence from $\omega / \Omega_{\text {Max }}$.

[21] T. Valla, et al., Science 258, 2110 (1999). 